\title{
Cultivation of Escherichia coli with mixtures of 3-phenylpropionic acid and glucose: Dynamics of growth and substrate consumption
}

\author{
K. Kovářová, A. Käch, V. Chaloupka \& T. Egli* \\ Swiss Federal Institute for Environmental Science and Technology (EAWAG), Swiss Federal Institute of Technology \\ (ETH), Überlandstrasse 133, CH-8600 Dübendorf, Switzerland (* Corresponding author, email: egli@eawag.ch)
}

Accepted 2 December 1996

Key words: dynamics, Escherichia coli, glucose, linear growth, mixed substrate, 3-phenylpropionic acid, pulse

\begin{abstract}
In technical as well as natural ecosystems, pollutants are often mineralised in the presence of easily degradable carbon sources. A laboratory model system consisting of Escherichia coli ML 30 growing with mixtures of 3phenylpropionic acid (3ppa, 'pollutant') and glucose (easily degradable substrate) was investigated in batch and carbon-limited continuous culture. Untypically, a linear growth pattern was observed during batch cultivation with $3 \mathrm{ppa}$ as the only carbon/energy source. When exposed to mixtures of both substrates in batch culture, E. coli utilised the two compounds sequentially. However, 3ppa and glucose were consumed simultaneously in continuous culture. Whereas a pulse of excess glucose to a batch culture growing with $3 p p a$ led to the repression of 3 ppa utilisation, an excess of glucose added into continuous culture did not inhibit the utilisation of 3 ppa. During continuous cultivation the 3ppa-degrading enzyme system operated close to saturation.
\end{abstract}

\section{Nomenclature}

\begin{tabular}{lll} 
3ppa & 3-phenylpropionic acid & \\
$D$ & dilution rate (specific growth rate in chemostat) & $\mathrm{h}^{-1}$ \\
DOC & dissolved organic carbon & $\mathrm{mg} \mathrm{L}^{-1}$ \\
DW & dry weight & $\mathrm{mg} \mathrm{L}^{-1}$ \\
$\mu_{\max }$ & maximum specific growth rate & $\mathrm{h}^{-1}$ \\
& & \\
\hline
\end{tabular}

\section{Introduction}

In natural as well as engineered environments, microorganisms most probably utilise and grow with many different carbon compounds at the same time (a phenomenon that has been referred to as "mixed substrate growth", e.g., Egli 1995; Harder \& Dijkhuizen 1982; Matin 1979). Among the many different naturally available carbon substrates, sugars and aromatic hydrocarbons certainly belong to those chemical structure units that are most abundant in nature (Münster 1993).

Whereas the metabolic pathways of sugars in Escherichia coli have been well studied (reviewed in, e.g., Lendenmann \& Egli 1995; Lengeler 1993; Neidhart et al. 1987), there is so far little known on the catabolism of aromatics. Although many different microorganisms are capable of degrading aromatic compounds, most of the present knowledge on aromatic catabolism has come from studies of bacteria that belong to the genus Pseudomonas (e.g., Smith 1990 ). The ability of $E$. coli to degrade aromatic compounds to completion was first reported in 1980 (Cooper \& Skinner 1980). In particular the catabolism of 3-phenylpropionic acid (3ppa; chemical structure see Figure 1) was investigated by Burlingame \& Chapman (1983), and a pathway for the degradation of 3ppa to succinate, acetaldehyde, and pyruvate was proposed. The reaction sequence was initiated by a dioxygenase attack at the aromatic ring, and the meta-ring cleavage occurred before the side chain degradation. This catabolic pathway in $E$. coli is similar to that observed earlier in Acinetobacter sp. (Dagley et al. 1965). To date only, some genes of the 3ppa degradative pathway have been cloned (Bugg 1993), and an E. coli aromatic hydrolase with a broad substrate range, which is involved in the degradation of 4-hydroxyphenylacetic 
acid (unfortunately, there is no reference to 3ppa specificity) has been characterised by Prieto et al. (1993).

The existing concepts on microbial growth kinetics are based on the assumption that single substrates are utilised (e.g., Monod 1942) despite the fact that the microorganisms in nature grow with substrate mixtures and under conditions of changing substrate availability - both with respect to the concentration and the presence of particular substrates. Therefore, it is essential to extend such kinetic models to mixed substrate growth, especially for the case of the degradation of pollutants in the presence of mixtures of natural substrates. These particular aspects have been investigated in three parallel studies using a model system consisting of $E$. coli ML 30 growing with mixtures of glucose (here, easily degradable substrate) and 3ppa ('pollutant'; Kovárová et al. 1996a,b, and this study). Using this experimental system, we were able to re-examine the tentative model proposed for utilisation of mixtures of sugars (Egli et al. 1993; Lendenmann et al. 1996) for two carbon substrates that are quite different with respect to the metabolic pathways involved in their utilisation and the regulation of enzyme expression. Here, a detailed investigation of the growth dynamics of $E$. coli with mixtures of $3 \mathrm{ppa}$ and glucose is presented.

\section{Materials and methods}

\section{Medium and culture conditions}

Escherichia coli ML 30 (DSM 1329) was grown at $37^{\circ} \mathrm{C}$ in both batch and continuous culture. The mineral medium was supplemented with either $3 \mathrm{ppa}$, or with mixtures of 3ppa (Fluka) and glucose (Merck) as the only sources of carbon and energy. Details on the medium composition and cultivation conditions were described previously (Senn et al. 1994).

\section{Analytical procedures}

\section{Glucose analysis}

The glucose concentrations in batch cultures and during the pulse experiments (where concentrations were in the $\mathrm{mg} \mathrm{L}^{-1}$ range) were measured enzymatically using the GOD-Period ${ }^{\circledR}$ method (Boehringer Mannheim, Germany). The samples were analysed at $420 \mathrm{~nm}$ (instead of $560 \mathrm{~nm}$ as recommended by the manufacturer). This modification allows a higher sensitivity (detection limit ca. $1-2 \mathrm{mg} \mathrm{L}^{-1}$ of glucose).
Residual concentrations of glucose in continuous culture (in the $\mu \mathrm{g} \mathrm{L}^{-1}$ concentration range) were analysed by HPLC according to Senn et al. (1994).

\section{3ppa analysis}

The samples were immediately filtered after they were withdrawn from the bioreactor or batch culture, and the $\mathrm{pH}$ was adjusted to 3 by adding concentrated $\mathrm{HCl}$. The 3ppa-concentrations were analysed by a standard HPLC application (Waters- Millipore 625LC; Waters 712 WISP injector, 50 to $150 \mu \mathrm{L}$ injection volumes used) with UV detection at $206 \mathrm{~nm}$ (Waters 991 photodiode array detector). The reverse-phase separation was achieved on a Nova Pak C-18 column (WatersMillipore) by applying a 1:1 mixture of $50 \mathrm{~mm} \mathrm{H}_{3} \mathrm{PO}_{4}$ and methanol ( $\mathrm{pH}$ adjusted to 3.1) as the eluent with a flow rate of $0.6 \mathrm{ml} \mathrm{min}{ }^{-1}$. The detection limit was ca. $0.01 \mathrm{mg} \mathrm{L}^{-1}$ of 3 ppa.

\section{Acetate analysis}

The concentration of acetate was measured by ionexclusion chromatography as described by Schneider et al. (1988).

\section{Dissolved organic carbon (DOC)}

DOC concentrations were measured in the filterate $(0.2 \mu \mathrm{m}$ polycarbonate membrane filter; Nuclepore, U.S.A.) after acidification with $\mathrm{HCl}$ using a Tocor 2 analyser (Maihak, Germany).

\section{Biomass determination}

Biomass was measured as dry weight (DW) by filtration through a $0.2 \mu \mathrm{m}$ pore size polycarbonate membrane filter (Nuclepore). Cells collected on filters (usually from of $100 \mathrm{~mL}$ cell suspension) were washed with distilled water, and filters were dried at $105^{\circ} \mathrm{C}$ to constant weight. Optical density was determined in 5-, 2-, or $1-\mathrm{cm}$ cuvettes at $546 \mathrm{~nm}$ with a Uvikon $860 \mathrm{spec}-$ trophotometer (Kontron, Switzerland).

\section{Specific oxygen uptake rates}

A sample of culture liquid $(12 \mathrm{~mL})$ was collected directly from the chemostat, centrifuged, washed twice with mineral medium, and resuspended in $3 \mathrm{~mL}$ of mineral medium (Senn et al. 1994). The samples taken from batch cultures were also washed two times, but resuspended in mineral medium to the original volume. The 3ppa- or glucose- stimulated oxygen uptake rate 


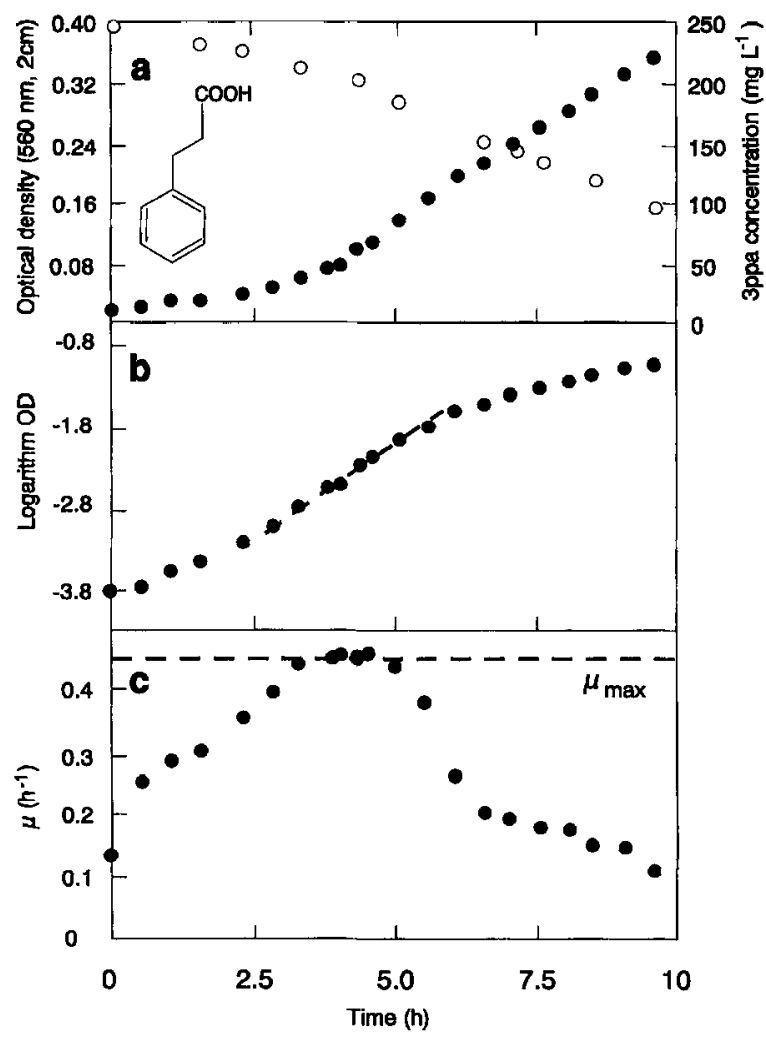

Figure 1. Typical linear batch growth curve for E. coli growing with 3 ppa (200 $\mathrm{mg} \mathrm{L}^{-1}$ of $\mathrm{C}$ ) as the sole source of carbon and energy. a - - biomass concentration measured as optical density, - $\mathrm{O}$ - 3ppa concentration; $b$ - logarithm of optical density as function of time, the dashed line indicates the 'nearly' linear part of this curve; $c$ - the specific growth rate as function of time, the maximum of this curve was referred to as a $\mu_{\max }$ of $0.45 \mathrm{~h}^{-1}$, which is indicated by the dashed line.

was recorded at $37^{\circ} \mathrm{C}$ in a Clark-type oxygen probe (Rank Brothers). The total volume of the assay was 3 $\mathrm{mL}$, consisting of $2.8 \mathrm{~mL}$ cell suspension of a known biomass concentration and $0.2 \mathrm{~mL}$ of $0.1 \mathrm{M}$ glucose or 3 ppa solution. The deviation of our measurements was about 10 to $20 \mathrm{nmol} \mathrm{O}_{2} \mathrm{~min}^{-1}(\mathrm{mg} \mathrm{DW})^{-1}$.

\section{The rate of linear growth}

The maximum specific growth rate is conventionally determined from the exponential phase of a growth curve. Untypically in our case, no exponential growth was observed over an extended period of time, and the initial 'exponential growth' was quickly followed by a linear growth phase. Because during the linear growth the growth rate changes with the time, we determined the $\mu=\frac{d x}{d t} \cdot \frac{1}{x}$ as a function of time. Here, the 'true' $\mu_{\max }$ was determined as the maximum of the $\mu(t)$ function; the approach is explained in Figure 1.

\section{Results \\ Growth with 3ppa as sole carbon and energy source in batch culture}

When $E$. coli ML 30 was grown in batch culture with 3ppa as the sole carbon and energy source, no distinct phase of exponential growth was observed, but the cells grew linearly (Figure 1). This did not change, although the cells were transferred repeatedly (eight times) from one batch culture to the next, where they always were supplied with $200 \mathrm{mg} \mathrm{L}^{-1}$ of 3 ppa carbon as the only carbon/energy source. In this way they all grew over more than 100 hours (i.e., ca. 50 generations) at an average maximum specific growth rate of $0.43 \pm 0.05 h^{-1}$. Although the cells grew linearly, 3ppa was completely utilised and decreased from an initial concentration of $248 \mathrm{mg} \mathrm{L}^{-1} \cong 180 \mathrm{mg} \mathrm{L}^{-1}$ of carbon from 3ppa) to concentrations $\leq 1 \mathrm{mg} \mathrm{L}^{-1}$ and final DOC concentrations in the medium of about $40 \mathrm{mg}$ $\mathrm{L}^{-1}$. (of the $40 \mathrm{mg} \mathrm{L}^{-1}, 28 \mathrm{mg} \mathrm{L}^{-1}$ of DOC originated from the non-utilisable EDTA present in the mineral medium.

The linear growth pattern did not significantly change when yeast extract (to a final concentration of $1 \mathrm{mg} \mathrm{L}^{-1}$ ), vitamins (for exact composition see Egli et al. 1988) or trace elements (supplied in the fresh mineral medium) were added to growing cells. However, the growth rate increased (but remained linear) when sterile filtered culture supernatant from a chemostat fed with a mixture of glucose and 3ppa was added to a culture growing in batch mode (Figure 2). Furthermore, no significant difference in the growth curves with 100,200 , and $400 \mathrm{mg} \mathrm{L}^{-1}$ of 3 ppa-carbon was observed, indicating no toxic effects of 3ppa within the tested concentration range. The growth yield was $1.13 \pm 0.07 \mathrm{mg} \mathrm{DW}$ (mg carbon) $)^{-1}$.

\section{Growth with mixtures of 3ppa and glucose in batch culture}

To obtain information concerning the regulation of the utilisation of $3 \mathrm{ppa}$ in the presence of alternative easily degradable carbon sources, the bacterium was grown in batch culture with mixtures of 3ppa and glucose (Figure 3). In these experiments the total carbon concentration was kept constant at $200 \mathrm{mg} \mathrm{L}^{-1} \mathrm{DOC}$, and 


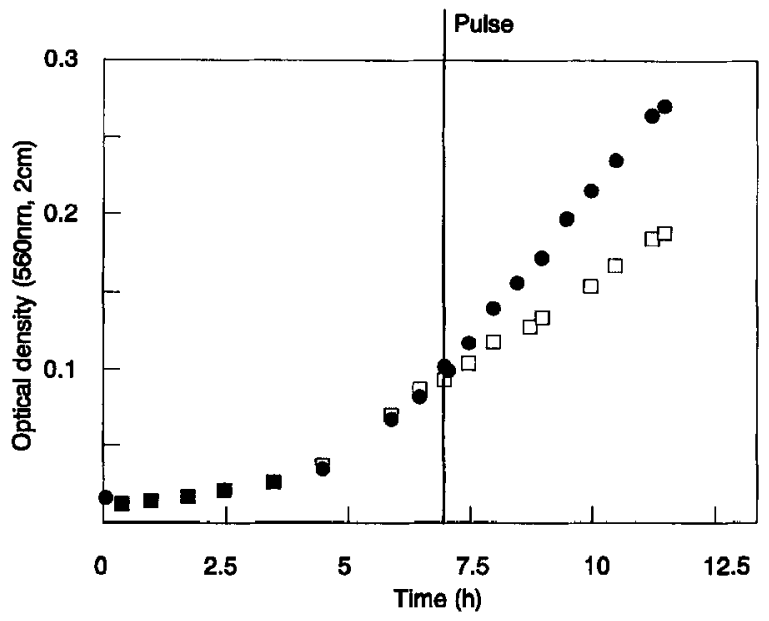

Figure 2. Growth of $E$. coli in batch cultures with 3 ppa as the sole carbon/energy source ( $200 \mathrm{~g} \mathrm{~L}^{-1}$ of 3 ppa carbon; total culture volume of $500 \mathrm{~mL}$ ) when pulsed with $20 \mathrm{~mL}$ of sterile filtrate from a chemostat culture grown with a mixture of $3 \mathrm{ppa}$ and glucose. As an inoculum cells grown in continuous culture with a 7:3 mixture of glucose:3ppa $(\mathrm{C}: \mathrm{C})$ were used; $O$ OD of pulsed culture, the pulse is indicated by a vertical line; $\square$ reference experiment.

only the proportion of the two substrates in the mixture was changed. The growth of $E$. coli followed a diauxic pattern (Figure 3 ). The utilisation of 3ppa was not detectable until glucose was exhausted (data for substrate concentrations not shown), and a lag-period of several hours was observed between the two growth phases. A typical example of the described growth pattern including the actual 3ppa and glucose concentrations is presented in Figure 4. Surprisingly, the specific growth rates observed in the two growth phases were influenced by the mixture composition (Figure 5). The maximum specific growth rate observed during growth with glucose decreased when increasing proportions of 3ppa were supplied in the initial mixture. Conversely, the maximum specific growth rate achieved in the 3ppa growth phase increased with increasing proportion of $3 \mathrm{ppa}$ in the mixture. The length of the lag-phase was dependent on the initial 3ppa:glucose ratio, and it increased with decreasing proportion of $3 \mathrm{ppa}$ in the mixture. Differently than in the previous experiments (Figures 1 and 2), the cells exponentially grown for more than 100 generations with glucose only (and never exposed to $3 \mathrm{ppa}$ ) in batch culture were used as the inoculum. It appears that such a different pre-treatment of the inoculum caused that 3 ppa in the initial phase of the batch culture to support exponential growth, in contrast to the data shown in Figures 1 and 2. During the later phase of batch growth, the cells clearly grew

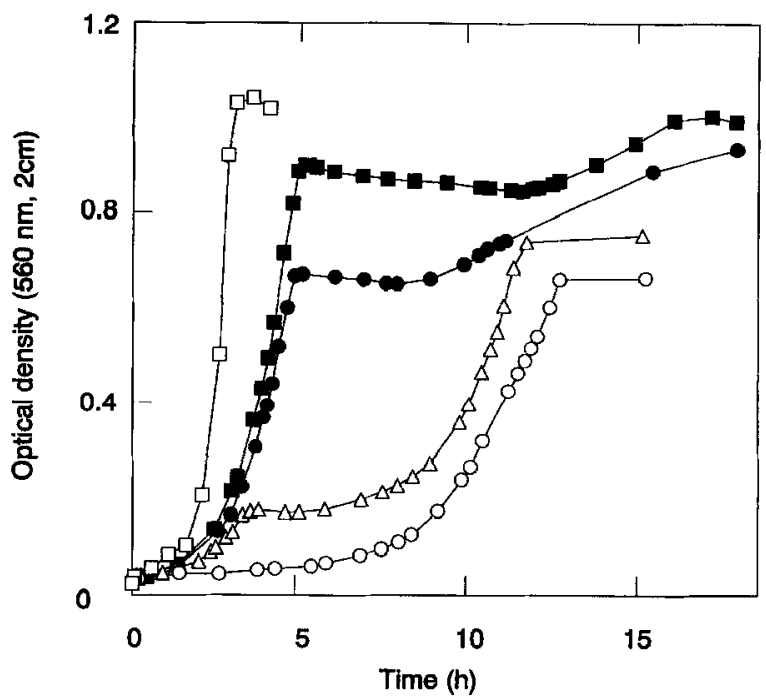

Figure 3. Growth of E. coli in batch culture with mixtures of 3ppa and glucose as the sole sources of carbon/energy (total carbon concentration of $200 \mathrm{mg} \mathrm{L}^{-1}$ ). As an inoculum cells pregrown in batch culture with glucose were used; the mixture composition is given in\% of carbon: $\square 100 \%$ of glucase; $\square$ mixture of $90 \%$ glucose and $10 \%$ 3ppa; mixture of $50 \%$ each substrate; $\triangle$ mixture of $10 \%$ of glucose and $90 \%$ of $3 \mathrm{ppa} ; 100 \%$ of $3 \mathrm{ppa}$.

linearly. (However, it is difficult to statistically distinguish the point at which the culture changes from an exponential to a linear growth behaviour.) Additionally, linear growth was observed when the cells were grown in batch culture with a mixture of 3 ppa and glucose (Figure 3); the standard deviation of the $\mu_{\max }$ estimates being $0.05 \mathrm{~h}^{-1}$.

Similar experiments were performed with mixtures of 3ppa and acetate. The utilisation patterns for acetate and 3ppa, as well as the DOC balance, gave strong evidence that $3 \mathrm{ppa}$ was not utilised before acetate was exhausted. However, no lag-phase was detected in the growth curve (data not shown).

\section{The response to changing substrate availability and its effect on the utilisation of $3 p p a$}

In both natural and technical environments, microorganisms are often exposed to fluctuating substrate availability. A 'new' substrate (pollutant) can be introduced into such a system either pulswise or more or less continuously over a longer time period. Here, 'simple' pulse and transient experiments were carried out as a contribution to the understanding of the complex regulation patterns involved in the utilisation of substrate mixtures when the substrate availability is changing. 


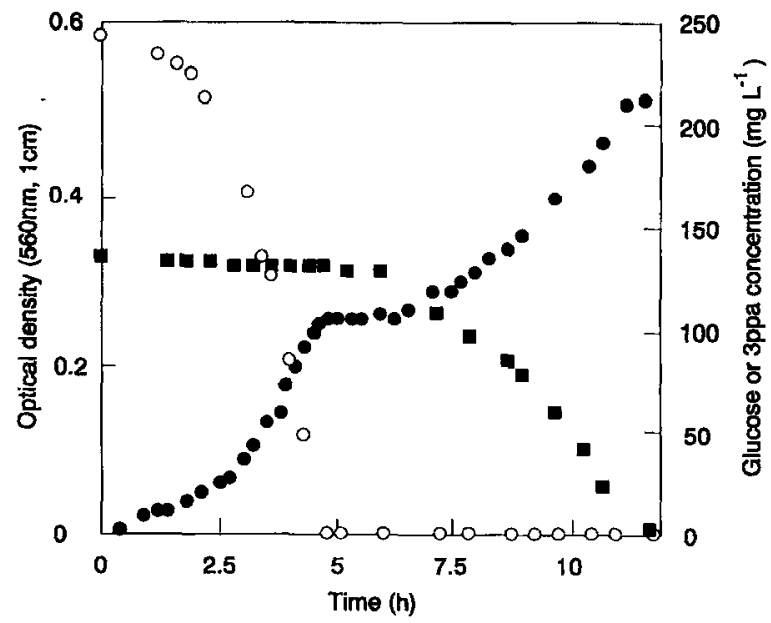

Figure 4. Batch growth of $E$. coli with a $1: 1\left(\mathrm{C}: \mathrm{C}, 100 \mathrm{mg} \mathrm{L}^{-1}\right.$ of carbon each) mixture of $3 \mathrm{ppa}$ and glucose. (Coptical density; $O$ glucose concentration; 3 ppa concentration).

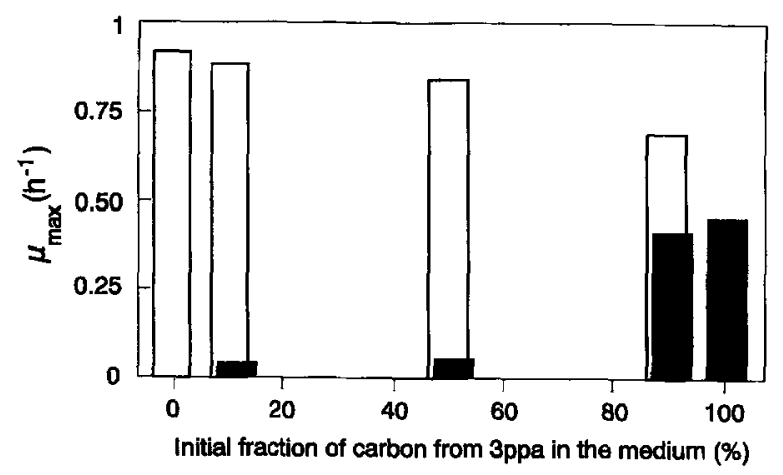

Figure 5. Maximum specific growth rates during growth of $E$, coli with mixtures of $3 \mathrm{ppa}$ and glucose in batch culture, as a function of the fraction of carbon from 3ppa in medium feed (for the growth curves see Figure 3 ; the standard deviation of the $\mu_{\max }$ estimates being $0.05 \mathrm{~h}^{-1}$ ). Open column - $\mu_{\max }$ determined during the growth phase on glucose; black column - $\mu_{\max }$ determined during the growth phase on 3ppa.

\section{Pulse of glucose to batch culture growing with 3ppa}

To investigate in more detail to what extent the $3 \mathrm{ppa}$ utilisation can be inhibited by glucose, $E$. coli was grown in batch mode with a mixture of 3ppa and glucose (initial concentrations being $100 \mathrm{mg} \mathrm{L}^{-1}$ of carbon from glucose and $200 \mathrm{mg} \mathrm{L}^{-1}$ of carbon from 3ppa). As an inoculum, cells from continuous culture growing with a 3:7 (C:C) mixture of 3ppa and glucose were used. Although the cells (when washed) exhibited 3ppa-oxidising capacity (Figure 6c), no apparent mixed substrate utilisation was detected (Figure 6a, d). When glucose was exhausted, the cells started to grow with 3ppa as the sole carbon/energy substrate after a short lag. An addition of excess glucose (to a final concentration in the culture medium of $100 \mathrm{mg}$ $\mathrm{L}^{-1}$ of carbon) during the 3ppa growth phase transiently repressed the utilisation of 3 ppa (Figure 6). The specific 3ppa-stimulated oxygen uptake rate increased when 3ppa was utilised and decreased during glucose growth phases (Figure 6c). An opposite pattern was observed for glucose-stimulated specific oxygen consumption rates (Figure 6b).

\section{Pulse of glucose to a continuous culture growing with 3ppa and glucose}

In contrast to batch culture, the two substrates were utilised simultaneously in carbon-limited continuous culture (see also Kovářová et al. 1996a). In order to study the effect of glucose on the utilisation of $3 \mathrm{ppa}$ in continuous culture, cells growing at $D=0.6 \mathrm{~h}^{-1}$ with a $7: 3(\mathrm{C}: \mathrm{C})$ mixture of glucose and 3ppa (100 $\mathrm{mg} \mathrm{L}^{-1}$ of glucose and $23.8 \mathrm{mg} \mathrm{L}^{-1}$ of $3 \mathrm{ppa}$ ) were pulsed with an excess of glucose (Figure 7). Immediately after the pulse, the glucose concentration in the bioreactor was $200 \mathrm{mg} \mathrm{L}^{-1}$ (i.e., $80 \mathrm{mg} \mathrm{L}^{-1}$ of $\mathrm{C}$, which is a comparable concentration range as that used in the batch experiments shown above). The biomass concentration, as well as the substrate concentrations and the specific oxygen consumption rates stimulated by glucose or $3 \mathrm{ppa}$, were measured as function of time (Figure 7). After the pulse, glucose concentration started to decrease (to final concentration of $<1 \mathrm{mg} \mathrm{L}^{-1}$ of glucose), and at the same time the biomass concentration started to increase. However, the utilisation of 3ppa, as indicated by the residual 3ppa concentrations in the culture, was not repressed (Figure 7a), and only a transient increase of 3ppa concentration from $300 \mu \mathrm{g} \mathrm{L}^{-1}$ (steady-state value prior the pulse) up to $1000 \mu \mathrm{g} \mathrm{L}^{-1}$ (peak concentration) was measured. Nevertheless, after the pulse, the 3ppa-stimulated specific oxygen consumption rates decreased until glucose was exhausted. After the exhaustion of glucose, the excess biomass resulting from the pulse started to wash-out from the bioreactor, and the 3ppa-stimulated oxygen consumption rates reached again the initial steadystate level of $88 \pm 15 \mathrm{nmol} \mathrm{O} \mathrm{O}_{2} \mathrm{~min}^{-1}(\mathrm{mg} \mathrm{DW})^{-1}$. During the whole experiment, the glucose- stimulated specific oxygen consumption rates remained constant at $167 \pm 18 \mathrm{nmol} \mathrm{O}_{2} \mathrm{~min}^{-1}(\mathrm{mg} \mathrm{DW})^{-1}$ (Figure $7 \mathrm{~b}$ ). 


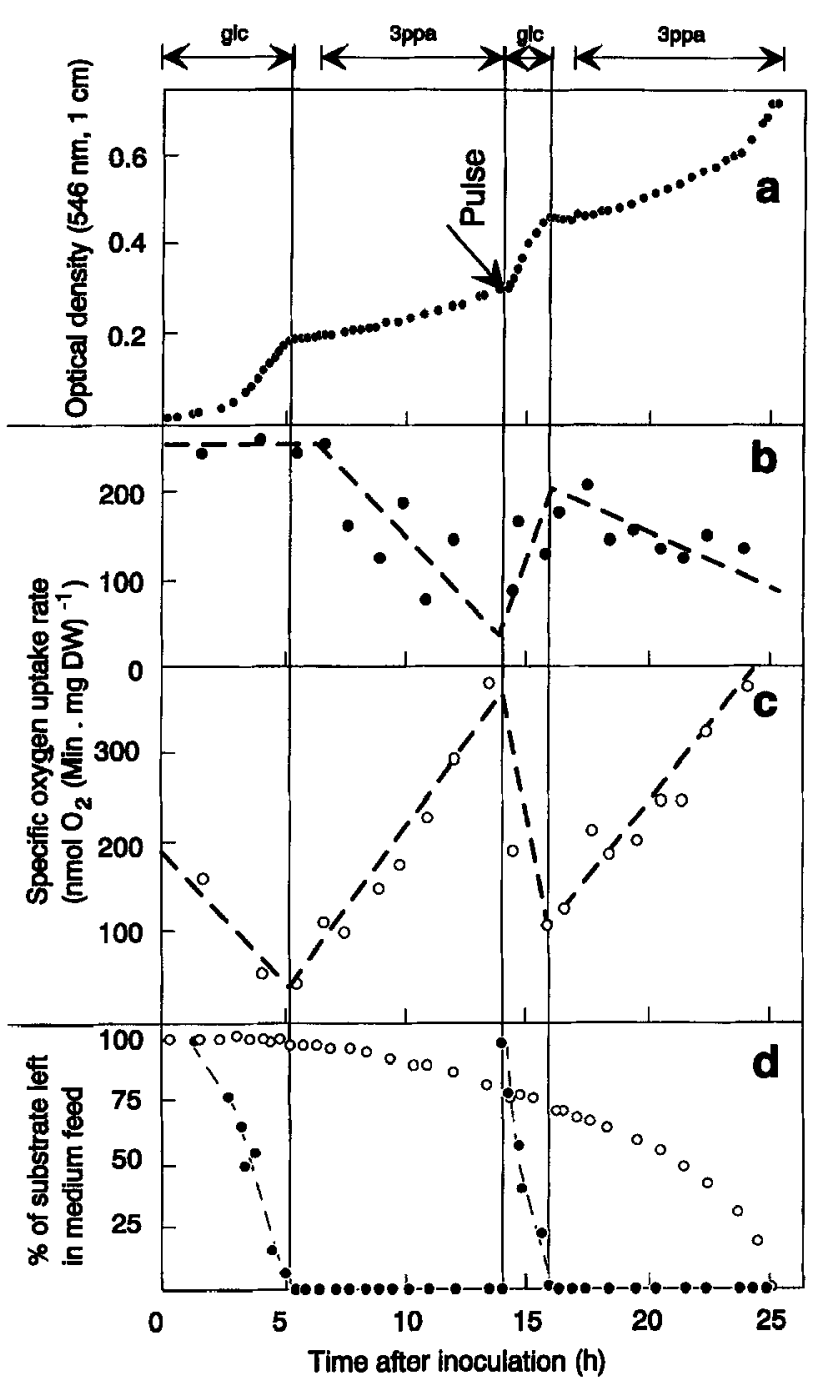

Figure 6. Pulse of excess of glucose into a batch culture growing with a mixture of glucose and $3 \mathrm{ppa}$. The initial mixture contained $100 \mathrm{mg} \mathrm{L}^{-1}$ of glucose carbon and $200 \mathrm{mg} \mathrm{L}^{-1}$ carbon from 3ppa; after ca. 840 minutes of cultivation glucose was pulsed (final concentration of $100 \mathrm{mg} \mathrm{L}^{-1}$ of glucose carbon) to the culture in the $3 \mathrm{ppa} / \mathrm{grow} t \mathrm{~h}$ phase; the arrows indicate the growth phases either with glucose or 3ppa; a - growth curve; b, c - glucose (0) or 3ppa (O) stimulated specific oxygen consumption rate; $d-\%$ of the initial substrate concentration left, - - glucose, $\bigcirc-3$ ppa.

\section{Transient shift from glucose to a mixture of glucose and 3ppa}

To investigate how fast $E$. coli can respond to a sudden availability 3 ppa, the cells were grown with glucose (100 $\mathrm{mg} \mathrm{L}^{-1}$ ) as the only carbon/energy source until a steady-state was reached. Then inlet medium was shifted to one containing the same concentration of glucose and, additionally, 3ppa. The mixture compo-

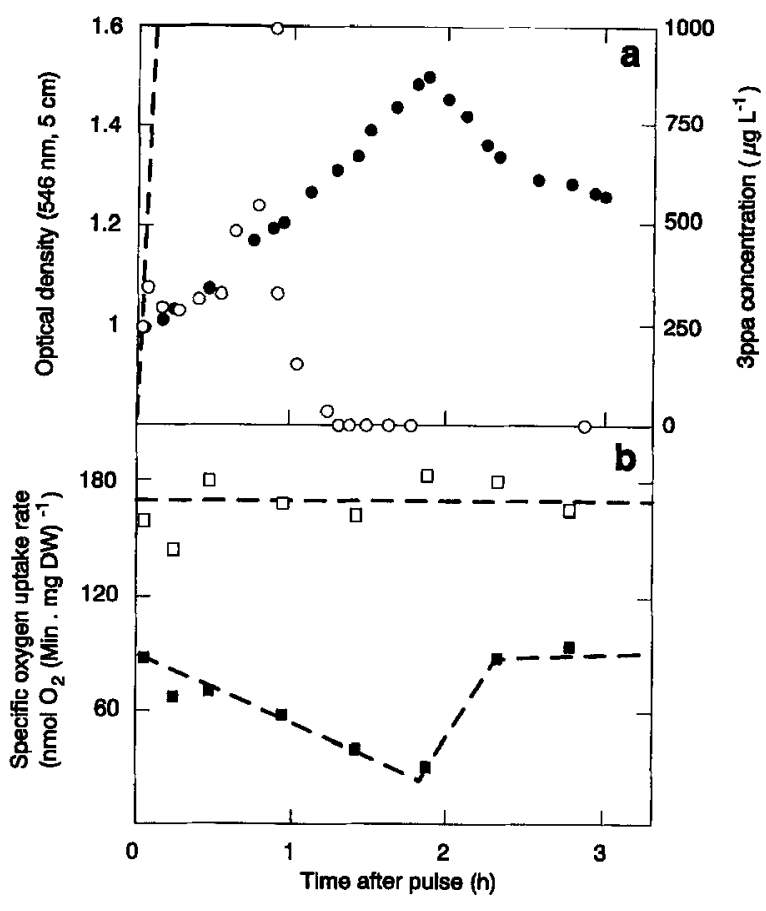

Figure 7. Pulse of excess of glucose into a continuous culture of E. coli growing with a 3:7 (C:C) mixture of 3ppa and glucose. a - $O$ - biomass concentration followed as optical density, 3 ppa concentration, the dashed line indicates the wash-in of $3 \mathrm{ppa}$ assuming that the utilisation of 3 ppa had stopped immediately after the pulse; b - glucose $(\square$ ) or 3ppa $\square$ ) stimulated specific oxygen consumption rate.

sition was $7: 3$ glucose:3ppa (C:C). This culture was during its 'history' transiently exposed to 3ppa for the second time; i.e., the culture was first grown with glucose for 260 generations, then it was cultivated with a mixture of 3ppa and glucose (590 generations), which was followed by a second period of growth with glucose only (234 generations). Then the above described experiment was performed. During the first medium shift, the steady-state with respect to biomass concentration was required two to three times more time then during the 3ppa-transient described above.

Similar to the pulse experiment in continuous culture, the glucose-stimulated specific oxygen consumption rate remained constant at $178 \pm 20 \mathrm{nmol} \mathrm{O}_{2} \mathrm{~min}^{-1}$ (mg DW) $^{-1}$ (Figure 8a). Approximately 1 hour was required until a stable 3 ppa-stimulated specific oxygen consumption rate of $80 \pm 15 \mathrm{nmol} \mathrm{O}_{2} \mathrm{~min}^{-1}$ (mg DW) ${ }^{-1}$ was achieved (note, the sample preparation, during which the culture remains in contact with $3 \mathrm{ppa}$, takes approximately 10 minutes). During this time period (see point 1 , Figure $8 \mathrm{~b}$ ), only $30 \%$ of the 3 ppa con- 


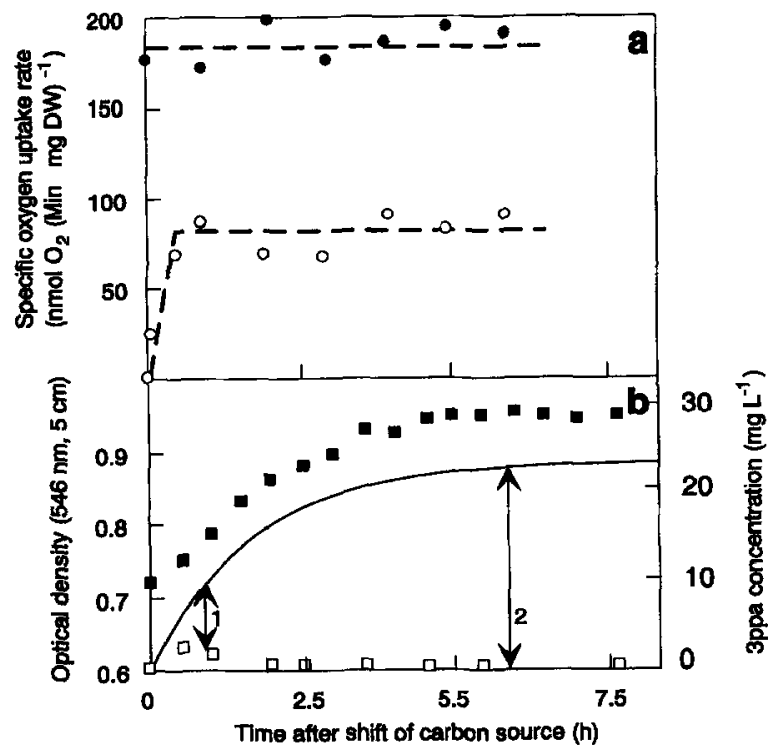

Figure 8. Transient behaviour of $E$. coli grown in carbon-limited continuous culture after switching the feed from medium containing glucose $\left(100 \mathrm{mg} \mathrm{L}^{-1}\right)$ as the only carbon and energy source to one containing the same concentration of glucose plus additionally $30 \%$ of 3ppa-carbon. a - glucose (O) or 3ppa (O) stimulated specific oxygen consumption rate; $b-\square$ - biomass concentration; $\square$ - actual 3ppa concentration, the curve indicates the theoretical wash-in of 3 ppa from the new medium feed; the arrows indicate the effectively utilised 3ppa concentration at the particular time point $(1-8 \mathrm{mg}$ $\mathrm{L}^{-1}, 2$ - $22 \mathrm{mg} \mathrm{L}^{-1}$ ).

centration in the medium feed was effectively washedin into the bioreactor and utilised (the actual $3 \mathrm{ppa}$ concentrations were always $<3 \mathrm{mg} \mathrm{L}^{-1}$ ). However, biomass concentration was still increasing due to the utilisation of the inflowing 3ppa (Figure 8b). Because the cells exhibited the same 3ppa-stimulated specific oxygen consumption rate $\left(80 \pm 15 \mathrm{nmol} \mathrm{O}_{2} \mathrm{~min}^{-1}\right.$ (mg DW) ${ }^{-1}$ ) whether 8 or $22 \mathrm{mg} \mathrm{L}^{-1}$ of 3 ppa were effectively utilised (Figure 8b), they temporarily had a 'spare capacity' for 3 ppa utilisation.

\section{Discussion}

\section{The phenomenon of linear growth in batch cultures}

Linear growth in batch cultures is known to be linked to conservative trace nutrient uptake (Brown et al. 1988), intracellular accumulation of the limiting substrate in any form (Novák et al. 1990), or inappropriate cultivation condition resulting in limitation by oxygen or substrate flux (i.e., constant rate of supply of oxygen or limiting substrate, reviewed by Brown et al. 1988).

Concerning the experimental observations during the growth of $E$. coli with 3 ppa as the sole source of carbon and energy, we can only speculate what is responsible for the linear growth pattern observed in these batch cultures. However, several factors can be excluded. For example, when the same mineral medium was supplemented with glucose or acetate instead of $3 \mathrm{ppa}$, no linear growth pattern was observed. Because, the cultures growing with 3ppa were extensively aerated and they did not show any response to the addition of trace elements (trace nutrients) or vitamins, only a limitation by intracellular metabolite accumulation or substrate transport might be considered. The cultures grew faster or nearly exponentially when sterile filtered culture supernatant from cells already growing with a mixture of 3 ppa and glucose was added (e.g., Figure 1).

It should be pointed out that the linear growth behaviour observed during batch cultivation with $3 \mathrm{ppa}$ as the sole substrate did not hamper growth in continuous culture. During continuous cultivation in the presence, or even without glucose (for more details on continuous cultivation see Kovárová et al. 1996a and $1996 b$ ), the cells grew exponentially (if $E$. coli was growing linearly in the chemostat the culture would have washed out). Therefore, only when estimating the $\mu_{\max }$ from batch growth data the linear growth pattern has to be considered (Figure 1). It was also observed that it is easier to start up a continuous culture with medium containing a mixture of 3 ppa and glucose than with 3ppa alone. Applying a 'too high' dilution rate in the initial phase after switching the culture to the chemostat mode with $3 \mathrm{ppa}$ as the sole carbon and energy substrate occasionally resulted in a wash-out.

Surprisingly, no 'spare capacity' with respect to 3 ppa consumption could be measured for cells growing in continuous culture (Kovářová et al. 1996b). The measured excess specific 3ppa consumption rate was virtually identical to that calculated from the dilution rate and medium composition. This indicates that we worked with a system that was close to saturation with respect to 3 ppa uptake. Therefore, even when an excess of 3ppa was added to the culture, it was impossible to detect any difference in the consumption rate. Consequently, a virtual 'spare capacity' with respect to 3 ppa consumption was observed transiently during the initial phase of the shift experiment (Figure 8). 
How is the 3ppa degradation regulated?

Our understanding of the regulation of 3ppa utilisation in mixtures with glucose remains at the phenomenological level that involves following the substrate concentrations and the substrate specific consumption capacities (i.e., excess rates). Here, we were mainly interested to what extent the utilisation of 3ppa can be inhibited by a second substrate.

In batch culture, the utilisation of 3ppa was immediately repressed by glucose, regardless of whether glucose was present in the initial substrate mixture or was pulsed to cells growing with 3ppa alone. Surprisingly, pulsing cells growing with 3 ppa in continuous culture showed quite a different response (Figures 7 and 8). Although the amount of glucose added resulted in comparable concentrations in both experiments, the pulse of excess glucose to continuous culture did not inhibit the utilisation of 3ppa. The effect of glucose was only visible as a transient reduction in the 3ppastimulated oxygen uptake rate. This observations raises the question of what is the difference in the regulation of these two systems.

One explanation might be that in continuous culture - in contrast to batch growing cells - the glucose uptake system did not operate so close to saturation. A 'spare capacity' to take up glucose was always measured under steady-state growth conditions (Kovárová et al. 1996b, Lendenmann \& Egli 1995). However, within the exponential growth phase in batch culture with glucose, the cells are supposed to work close to saturation (shown in Figure 6 for the first growth phase). Due to this 'spare capacity' the cells grown in continuous culture might be able to utilise the excess glucose without visibly affecting the utilisation of $3 \mathrm{ppa}$.

\section{Acknowledgments}

We are grateful to Albert Tien for correcting the English and for advice concerning the measurements of oxygen uptake rates. Furthermore, the authors are indebted to Christian Zipper and Stefano Simoni for advice concerning the 3ppa analysis, Hansueli Weilenmann and Thomas Fleischmann for skilled technical assistance during DOC measurements.

\section{References}

Brown DE, Gaddum RN \& McAvoy A (1988) Linear growth in batch culture caused by conservative trace nutrient limitation. Biotechnol. Letters 10: 525-530

Bugg TDH (1993) Overproduction, purification and properties of 2,3-dihydroxyphenylpropionate 1,2-dioxygenase from Escherichia coli. Biochim. Biophys. Acta 1202: 258-264

Burlingame R \& Chapman PJ (1983) Catabolism of phenylpropionic acid and its 3-hydroxy derivate by Escherichia coli. J. Bacteriol. 155: 113-121

Cooper RA \& Skinner MA (1980) Catabolism of 3- and 4hydroxyphenylacetate by the 3,4- dihydroxyphenylacetate pathway in Escherichia coli. J. Bacteriol. 143: 302-306

Dagley S, Chapman PJ \& Gibson DT (1965) The metabolism of 3-phenylpropionic acid by an Achromobacter. Biochem. J. 95: $466-474$

Egli T (1995) The ecological and physiological significance of the growth of heterotrophic microorganisms with mixtures of substrates. In: Gwynfryn Jones J (Ed) Advances in Microbial Ecology, Vol 14 (pp 305-386). Plenum Press, New York

Egli T, Lendenmann U \& Snozzi M (1993) Kinetics of microbial growth with mixtures of carbon sources. Antonie van Leeuwenhoek 63: 289-298

Egli T, Weilenmann H-U, El-Banna T \& Auglin G (1988) Gramnegative, aerobic, nitrilotriacetate-utilising bacteria from wastewater and soil. Syst. Appl. Microbiol. 10: 297-305.

Harder W \& Dijkhuizen L (1982) Strategies of mixed substrate utilisation in microorganisms. Phil. Trans. R. Soc. Lond. B. 297 : $459-480$

Kovářová K, Käch A, Chaloupka V, Zehnder AJB \& Egli T (1996a) Cultivation of Escherichla coli with mixtures of 3phenylpropionic acid and glucose: steady-state growth kinetics. Submitted.

Kovářová K, Chaloupka V \& Egli T (1996b) Threshold substrate concentrations required for induction of the catabolic pathway of 3- phenylpropionic acid in Escherichia coli. Submitted.

Lendenmann U \& Egli T (1995) Is Escherichia coli growing in glucose-limited chemostat culture able to utilize other sugars without lag? Microbiology 141: 71-78

Lendenmann U, Snozzi M \& Egli T (1996) Kinetics of simultaneous utilisation of sugar mixtures by Escherichia coli in continuous culture. Appl. Environ. Microbiol. 62: 1493-1499

Lengeler J. W. (1993) Carbohydrate transport in bacteria under environmental conditions, a black box? Antonie van Lecuwenhoek 63: $275-288$

Matin A. (1979) Microbial regulatory mechanisms at low nutrient concentrations as studied in chemostat. In: Shilo M (Ed) Strategies of Microbial Life in Extreme Environments (pp 323-339). (Berlin: Dahlem Konferenzen, Verlag Chemie, Weinheim

Müster U (1993) Concentrations and fluxes of organic carbon substrates in the aquatic environment. Antonie van Leeuwenhoek 63 : 243-264

Neidhardt et al. (Eds.) (1987) Escherichia coli and Salmonella typhimurium. American Society for Microbiology, Washington, D.C.

Novák M, Sikyta B, Klener V \& Novotny C (1990) Linear growth in microbial cultures limited by accumulated substrate. Biotechnol. Letters 12: 247-252

Prieto MA, Perez-Aranda A \& Garcia JL (1993) Characterization of an Escherichia coli aromatic hydrolase with a broad substrate range. J. Bacteriol. 175: 2162-2167 
Schneider RP, Zürcher F, Egli T \& Hamer G (1988) Determination of nirilotriacetate in biological matrices using ion exclusion chromatography. Anal. Biochem. 173: 278-284

Senn H, Lendenmann U, Snozzi M, Hamer G \& Egli T (1994) The growth of Escherichia coli in Glucose- limited chemostat cultures: a reexamination of the kinetics. Biochim. Biophys. Acta 1201: $424-436$
Smith MR (1990) The biodegradation of aromatic hydrocarbons by bacteria. Biodegradation 1: 191-206 